\title{
Satisfacción laboral en una organización human care de la Bahía de Guayaquil
}

\section{Job satisfaction in a human care organization in the Guayaquil Bay}

Luis Antonio Álvarez Silva ${ }^{1}$, Lourdes Adriana López Rodríguez ${ }^{2}$, Nelly Elizabeth Silva Avilés ${ }^{3}$

INFORMACIÓN DEL

\section{ARTÍCULO}

Fecha de recepción: 19 de Marzo de 2019.

Fecha de aceptación: 16 de Junio de 2019

\section{Resumen}

Esta investigación tiene como finalidad dar a conocer los factores más importantes de la satisfacción laboral en los empleados de una empresa comercializadora de productos de cuidado personal en la zona mercantil de la bahía de Guayaquil. El diseño investigativo utilizado fue no experimental, tipo transversal. En su desarrollo, se utilizó el cuestionario de Satisfacción laboral S20/23 de Melía y Peiró; este, presentó un nivel de consistencia interna alta $(\alpha=0.90)$. El estudio tuvo una muestra por conveniencia de 201 empleados; el proceso en general se llevó a cabo en el año 2018. Se encontró que la participación en la toma de decisiones, la remuneración y las condiciones físicas son factores de insatisfacción laboral. Una de las conclusiones predominantes es que el sistema de compensaciones y las condiciones de trabajo influyen en la satisfacción de los trabajadores.

\section{Palabras Clave:}

Satisfacción laboral, factores organizacionales, trabajo formal, organización, talento humano, gestión humana.

\section{Clasificación JEL: J28.}

Universidad de Guayaquil. Directora Ejecutiva de Varez Consulting.

E-mail: nsilva@varezconsulting.com. Código ORCID

https://orcid.org/0000-0001-8620-3332
CITACIÓN: Álvarez Silva, L.A., López Rodríguez, L.A., \& Silva Avilés, N.E. (2019). Satisfacción laboral en una organización human care de la Bahía de Guayaquil. Podium, 35, 87-96. doi:10.31095/podium.2019.35.6

ENLACE DOI:

http://dx.doi.org/10.31095/podium.201 9.35 .6

\begin{abstract}
The aim of this research is to identify the most important factors of job satisfaction in employees of a human care company located in the Guayaquil Bay. The research design was not experimental and transversal. In its development, the Melia and Peiró's Work Satisfaction Questionnaire S20/23 was applied; this, presented a high internal consistency level $(\alpha=0.90)$. The study had a convenience sample of 201 employees collected in 2018. Participation in decision making, remuneration and physical conditions are factors of job dissatisfaction. One of the predominant conclusions is that the compensation system and the working conditions influence worker satisfaction.
\end{abstract}

Keywords:

Job satisfaction, organizational factors, formal work, organization, human talent, human management.

JEL Classification: J28. 


\section{Introducción}

En la última década, la gestión de recursos humanos ha venido evolucionando continuamente y ha sido de mucha relevancia para las organizaciones, ya que permite generar ventajas competitivas enfocadas en mejorar el desempeño de sus colaboradores (Arias y Justo, 2013). Consecuentemente, también se ha hecho conciencia de lo beneficioso que es establecer condiciones laborales de bienestar y potencializar la calidad de vida de los individuos (Chiang, Méndez y Sánchez, 2010). Por esta razón, se han generado estudios donde se analizan variables muy importantes para las organizaciones, como son: el estudio del clima laboral y la satisfacción en el trabajo. Estos aspectos contribuyen de manera sustancial a la administración del talento humano y al bienestar de los sujetos (Arias y Arias, 2014); los mismos se realizan por medio de levantamiento de información con herramientas y procesos confiables que concluyen en: la creación de esquemas de mejora de la calidad de vida laboral, la delimitación de sistemas de gestión de talento humano y la mejora de las estrategias hacia el desarrollo humano (Rodríguez, Retamal, Lizana, y Cornejo, 2011). Para Silva (1996), en algunas ocasiones las organizaciones propician proyectos de motivación, pero sin analizar previamente los elementos relevantes, tales como: el clima, la cultura; por ello, se tiene como efecto que dichos programas, fracasen o tengan un impacto no significativo. Por consiguiente, y considerando épocas contemporáneas, varios autores señalan que la relación entre la satisfacción laboral y el desempeño es más clara y directa, siendo la primera predictor de la segunda (Patterson, Warr y West, 2004). Además, se puede acotar que existe una correspondencia positiva y negativa entre ambas variables, ya que se estipula que los empleados más satisfechos son más productivos y rentables, siendo lo contrario, que personas insatisfechas presentan mayor índice de bajo desempeño, absentismo y rotación (Chiang y Ojeda, 2013).

Desde esta óptica, las organizaciones deben comprender que la satisfacción laboral se presenta de forma dual, es decir, como causa y como efecto (Griffin, Phillips, y Stanley, 2017), por ende, esta afecta a la motivación del trabajador. Además, entre ambas variables existe una relación indisoluble, ya que un colaborador satisfecho en su actividad laboral, está motivado para trabajar y se involucra más con su entorno de laboriosidad (Arias-Jiménez, 2007). Es por ello, que las organizaciones en general se vuelven escenarios sociales donde este fenómeno trasciende (Sánchez-Sellero, SánchezSellero, Cruz- González y SánchezSellero, 2014). En el caso de la organización objetivo, esta se dedica a la comercialización de productos de cuidado personal o human care, y está localizada en la zona mercantil denominada la bahía del centro de la ciudad de Guayaquil. Esta investigación se enfoca en analizar el nivel de satisfacción laboral de sus empleados. Por esta razón, el objetivo principal de 
esta investigación es conocer los factores más importantes de la satisfacción laboral, para lo cual se describirá de forma teórica el origen y evolución del término satisfacción laboral; además, se analizarán ciertas perspectivas laborales de la organización; y, a través de un estudio empírico, se establecerán resultados y conclusiones. Los resultados del presente estudio aportarán al desarrollo del conocimiento del tema, ya que, en la actualidad, la única manera de gestionar el cambio es analizando el desempeño y la situación de los trabajadores; ya que, con nuevos enfoques administrativos, el capital humano es esencial para el desarrollo organizacional (Alles, 2015).

\section{Revisión de literatura}

\section{Satisfacción Laboral}

En las últimas décadas, la satisfacción laboral ha sido objeto de estudio en variadas investigaciones. Según Greenberg y Baron (2003), esta se encuentra compuesta por varias dimensiones entre las que denotan: la remuneración, las diferentes tareas a realizar, las oportunidades de progresar y las relaciones interpersonales; estas variables, a su vez se encuentran ampliamente identificadas por el intercambio entre el individuo y las características del ambiente laboral (Hegney, Plank, y Parker, 2006). De acuerdo con el enfoque de los autores Salessi y Omar (2018), la satisfacción laboral se puede definir, como el modo de actuar que el trabajador muestra a su actividad, basándose en valores y afirmaciones que el colaborador desarrolla de su propia acción laboral y que probablemente intervendrán de manera significativa en su proceder.

Desde otro punto de vista, la satisfacción laboral es definida como el grupo de sentimientos benéficos y perjudiciales mediante los cuales los trabajadores consideran su empleo (Sánchez-Sellero y Sánchez-Sellero, 2018), produciéndose de forma positiva muchos beneficios como son: mantener una buena salud mental, productividad en la empresa, mejora en la rentabilidad $\mathrm{y}$ efectos negativos como lo son: el ausentismo, absentismo, el nivel de rotación, etc. Para Weinert (1985), la satisfacción laboral ha marcado su importancia en el mundo empresarial y el interés por el tema se debe al desarrollo de las teorías organizacionales, las cuales han evolucionado a través del tiempo (Temkin y Cruz, 2018).

Es importante destacar que existen características que se desarrollan de forma adjunta a la satisfacción en el trabajo, y que se las puede identificar como diferentes variables, ya sean de tipo organizacional, funcional o individual, como son: la retribución, la tarea, las interacciones personales, el entorno, la seguridad y salud ocupacional, entre otras. Con referencia al listado de variables anteriores, se pueden agrupar tres modelos asociados al concepto de satisfacción, tales como: modelos centrados en el sujeto, en el contexto y determinados por la interacción de ambos actores (Sánchez et al, 2014). 
Según los investigadores PlatánPérez, Martínez, y Hernández (2012), la satisfacción en el trabajo se arraiga a diferentes elementos, estos hacen referencia a las características personales tales como los rasgos personológicos, edad, dependientes económicos, escolaridad, particularidades del puesto, como el nivel de jerarquía, actividad, salario, prestaciones y a las características de las organizaciones, como la estructura, liderazgo, trabajo en equipo y apoyo grupal. En nuestro medio latinoamericano, existen estudios efectuados sobre satisfacción laboral, en la que los autores Peña, Olloqui y Aguilar (2013), concluyen que esta se encuentra relacionada con la remuneración y que la relación con los mandos superiores producen menos satisfacción. Del mismo modo, otra investigación sobre satisfacción en el trabajo, realizada por Manosalvas, Manosalvas y Quintero (2015), analizó que las causas de la satisfacción en el trabajo son congruentemente similares, y estas serían: condiciones materiales del trabajo, relaciones interpersonales en el trabajo y con directivos, remuneraciones, desarrollo, permanencia laboral $\mathrm{y}$ reconocimiento.

La organización en la que se realizó el estudio, tiene varias décadas de operación en el populoso sector comercial de la Bahía de Guayaquil; su misión es comercializar productos de cuidado personal a la comunidad, y su visión radica en ser una de las más exitosas en su ámbito.

Las características laborales de la compañía obedecen a un modelo mecanicista, en el cual la alineación de objetivos se orienta a la producción y los resultados; además, la toma de decisiones es exclusivamente de los directivos (Munch y García, 2017). La organización trata de cumplir todos sus indicadores de gestión con base a las reglamentaciones de las instituciones públicas que la auditan; por ello, la empresa cumple con el otorgamiento de contratos de trabajo (legales) a sus empleados, tomando como referencia el código de trabajo del Ecuador (Ministerio de Trabajo, 2005). Posee un organigrama dividido en dos áreas: área operativa y área administrativa; el área operativa comprende todo el proceso de insumos y procesos terminados, es decir, en ésta área operan los departamentos de comercialización y bodegaje. Mientras en el área administrativa se encuentran los departamentos de Gerencia, Recursos Humanos, Contabilidad, Logística y Legal. La compañía cuenta con 214 trabajadores, de los cuales la mayoría pertenece al área operativa.

\section{Metodología}

Esta investigación es de carácter no experimental y de tipo transversal; su enfoque es cuantitativo, ya que la utilidad de la herramienta y la presentación de los resultados permiten realizar un análisis numérico y objetivo. El alcance es descriptivo, y el fenómeno se abordó con un modelo de muestreo no probabilístico tipo intencional o también denominado por conveniencia, ya que la muestra depende de los criterios de los investigadores (Hernández, Fernández, y 
Baptista, 2014), por ello, sólo se consideró a los empleados que tenían alrededor de un año en labores activas, dato que fue corroborado con la última nómina del área de talento humano; esto representa el $94 \%$ de todos los empleados.

Se utilizó el cuestionario de satisfacción laboral S20/23, versión optimizada del cuestionario S4/82 (Meliá y Peiró, 1989), que contiene dimensiones de análisis que son de relevancia para esta investigación: la satisfacción relacionada con la supervisión, con el ambiente físico, con el salario, con el aspecto intrínseco y con el grupo de trabajo (Meliá y Peiró, 1998). El instrumento consta de 23 Ítems, cada uno posee siete (7) opciones de respuesta que permiten elegir las marcaciones partiendo de las nomenclaturas: 1: Muy Insatisfecho; 2: Bastante Insatisfecho; 3: Algo Insatisfecho; 4: Indiferente; 5: Algo Satisfecho; 6: Bastante Satisfecho, 7. Muy Satisfecho.

La sectorización del S20/23 fluctúa en cinco (5) factores que benefician su análisis, estos son: Factor 1, que hace referencia a la satisfacción producto de la supervisión, éste incluye a los ítems 13-14-15-16-17-18. Factor 2, analiza a la satisfacción con respecto a las condiciones físicas del trabajo, este incluye a los ítems 6-7-8-9-10. Factor 3, el cual hace reseña a la satisfacción relacionadas a la participación en toma de decisiones y prestaciones salariales, incluye a los ítems 4-11-12-22-23. Factor 4, identifica la satisfacción por condiciones intrínsecas, es decir a la percepción que el sujeto tiene de su propio trabajo, este incluye a los ítems 1-2-3-5 y finalmente el Factor 5, en referencia a la satisfacción por el reconocimiento personal, incluye a los ítems 19-20-21.

Una vez obtenida la aprobación de las gerencias y la comunicación organizacional del estudio, se procedió a presentar el cuestionario S20/23 a los trabajadores de la compañía en la sala principal, estos fueron divididos en bloques de 15 personas por cada área, con el fin de socializar cada punto y que ellos conozcan las características. El levantamiento total de la información se realizó en 21 días hábiles; y todo el proceso se realizó en el año 2018. Se obtuvo un coeficiente de consistencia interna Alfa de Cronbach $\alpha=0.90$. La validez del cuestionario se realizó a partir de un análisis factorial, el cual proporcionó un puntaje de 0.90 en su medida de Kaiser-Meyer-Olkin de adecuación de muestreo; la prueba de esfericidad de Barlett presentó un puntaje de 0.0 en su grado de significancia; $\mathrm{y}$, su promedio de comunalidades oscila desde 0.50 a 0.66 . La tabulación general y análisis descriptivo de los resultados se los realizó por medio del programa SPSS versión 22 y Microsoft Excel.

\section{Resultados}

La demografía de la población de estudio (género, educación, área de labor, instrucción y estado civil) se la puede apreciar en la Tabla 1. 
Tabla 1.

Características de la muestra

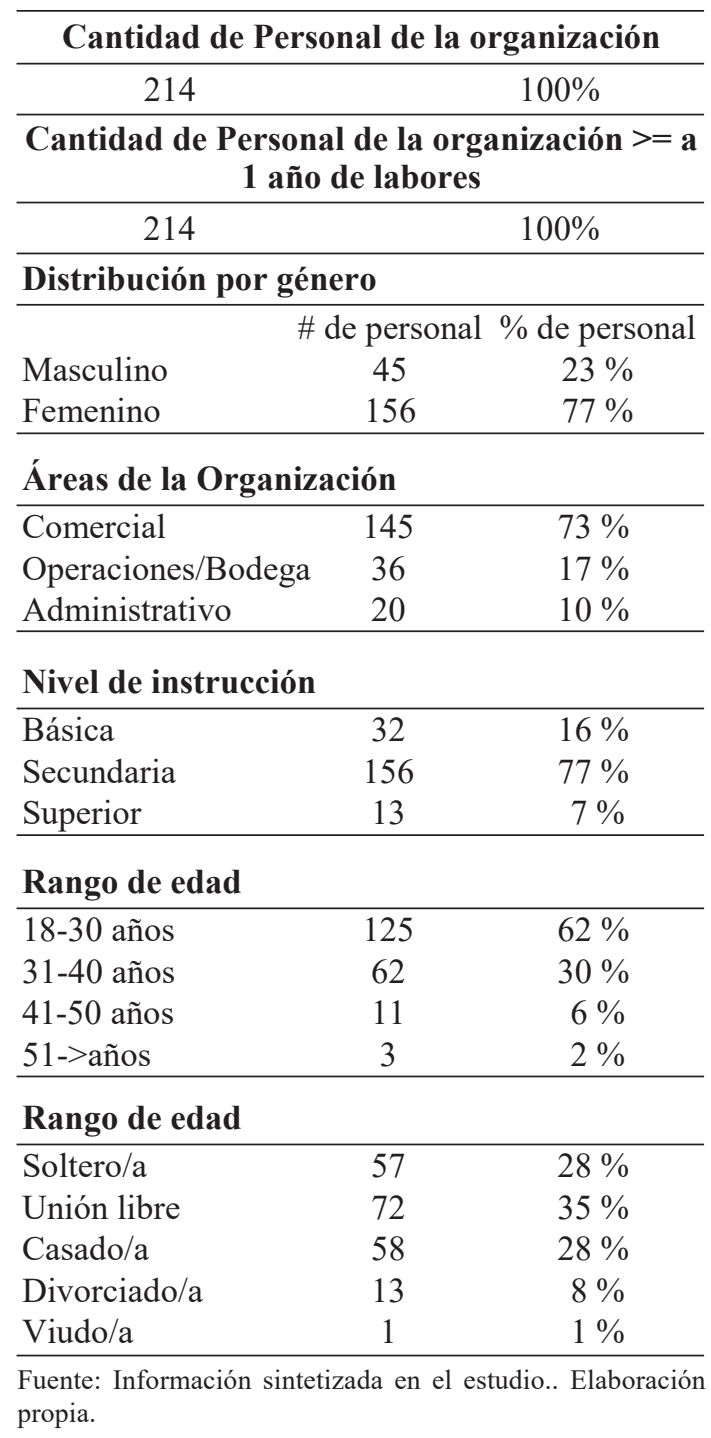

Factor 1: Se obtiene un 63.11\%; según el análisis, esto representa un grado de insatisfacción, el mismo que está relacionado con la característica del liderazgo que ejercen los mandos altos y medios. Factor 2: $64.90 \%$, esta cifra representa un nivel de insatisfacción; en este punto se establece que las condiciones de trabajo muestran relación con riesgos potenciales, tales como: accidentes de trabajo, enfermedades profesionales; por ende la importancia de reglamentación de seguridad y salud en el trabajo. Factor 3: 65.76\% de insatisfacción; la falta de prestaciones y la situación relacionada con el salario es la primera opción más alta de los resultados de factores; este indicador hace referencia a la relación entre funciones financieras y la justificación de las jornadas de trabajo, los pagos, afiliaciones, aseguramientos, multas, etc. Factor 4: $64.58 \%$ en nivel de insatisfacción, siendo este el penúltimo de los factores en calificarse; este resultado brinda información sobre la alineación del talento humano hacia las metas de la organización y la percepción laboral de los sujetos. El factor 5: 62.67\% de insatisfacción, este es el factor de menor porcentaje, sin embargo posee una alta significancia; el mismo hace referencia al tema de cohesión de personas y toma de decisiones entre los miembros de los grupos formales e informales. En la Tabla 2 se presenta un resumen de los resultados por factores.

Tabla 2.

Resultados por factores.sectoriales.

\begin{tabular}{ccccc}
\hline Factor 1 & Factor 2 & Factor 3 & Factor 4 & Factor 5 \\
$63.11 \%\left(I^{*}\right)$ & $64.90 \%$ ( I ) & $65.76 \%$ ( I ) & $64.58 \%$ ( I ) & $62.67 \%(\mathrm{I})$ \\
$36.89 \%\left(\mathrm{~S}^{*}\right)$ & $35.10 \%$ ( S ) & $34.24 \%$ ( S ) & $35.42 \%$ ( S ) & $37.33 \%$ ( S )
\end{tabular}

Fuente: Elaborado por los autores.

Ciertas compañías consideran que la rentabilidad económica es la razón de la existencia empresarial; sin embargo, aquella rentabilidad es sólo un producto con características de variabilidad; es por ello que organizaciones del siglo XXI tienen claro el escenario, la ganancia monetaria es el resultado de la satisfacción de los usuarios, la calidad en los procesos y sobre todo la satisfacción de sus trabajadores (Yohalmo, 2014). 
Dentro de la evidencia de investigaciones científicas, que hacen referencia a temas similares al presente estudio, existen investigaciones latinoamericanas que han utilizado la herramienta S20/23, para medir la satisfacción en el campo de trabajo; en estos estudios se muestra que el clima laboral influye en la satisfacción (Temkin y Cruz, 2018) y que la supervisión es un elemento clave en el desarrollo de los empleados (Cantón y Téllez, 2016). Los resultados de la investigación señalan que las dimensiones arrojaron un alto porcentaje de nivel de insastifaccion; haciendo ímpetu en las categorías de participación en la toma de decisiones, remuneración, condiciones de seguridad y salud laboral, desarrollo personal; ejemplo de ello es el alto porcentaje de insastifacción que se obtuvo en la categoría o factor remunerativo y de condiciones fisicas de trabajo. Esto, se podría relacionar con el aspecto de cultura organizacional. En relación a otros estudios de satisfaccion laboral, los factores de mayor incidencia se enfocan en la naturaleza de trabajo, las experiencias laborales, el tipo de puesto, entre otros (Cantón y Téllez, 2016). Por otra parte, las condiciones de trabajo y la estimulacion moral son los referentes mas importantes según estudios cuasiexperimentales de satisfacción a empleados (Álvarez, Guzmán, Noda, Álvarez, y Galcerán, 2016).

Por esta razón, la satisfacción laboral continúa siendo un estado psicológico, que se presenta por la exposición del sujeto a diferentes condiciones de trabajo, lo cual varía según la personalidad y las características de trabajo. Existen organizaciones que se preocupan por el bienestar, salud y deleite de sus colaboradores, normalmente esto acontece cuando existe una postura laboralmente estratégica, en la que el logro de la misión y alcance de la visión de la compañía se da en concordancia al desarrollo personal (Alles, 2015). De allí que poseer una cultura estratégica, comprende preocuparse por la satisfacción laboral como un componente que se debe medir. Sin embargo, algunas compañías sólo resumen el estudio de la satisfacción laboral en un informe de estudio de clima laboral básico.

\section{Conclusiones}

La satisfacción en el trabajo, es un elemento de suma importancia en las organizaciones, por ello su estudio y abordaje proporciona la percepción de los miembros de toda la compañía. Empresas con culturas emprendedoras, estratégicas y con una clara visión, aceptan que la complacencia de un trabajador es un logro en la empresa; por ello el bienestar se vuelve transversal. En esta investigación, la satisfacción se inclina a un nivel bajo, por lo que esto se lo podría considerar como insatisfacción a los factores que tienen mayor trascendencia, estos son: el sistema de compensaciones, las condiciones ambientales y psicológicas, y el desarrollo personal. Esto podría generar, como recomendación, una propuesta de mejora en el sistema de gestión de talento humano que puede devenir en progresos de mediano plazo a toda la organización. Además, como alterativa complementaria 
y específica, el área de gestión humana puede utilizar estos datos para implementar programas de salario emocional, análisis financiero de remuneración variable, creación del subsistema de seguridad e higiene en el trabajo, planes de carrera, presentación y seguimiento de un esquema de coaching a los directivos; todo esto, con sus respectivos indicadores de gestión, para así favorecer el proceso de bienestar de los miembros de la empresa.

Con respecto a la teorización y la cultura, uno de los elementos de mayor incidencia en la satisfacción es la condición Psicológica del sujeto; es decir, la percepción que él tiene sobre su trabajo (Zayas, Báez, Zayas, y Hernández, 2015); por ello, teóricamente la satisfacción en el trabajo es un tema latente e importante en psicología laboral y gestión del talento humano; pero culturalmente es un tema de poca preocupación; sin embargo, tiene varios enfoques disciplinarios y en algunos casos se lo estudia con otras variables organizacionales, tales como: la productividad, el coaching, la rotación, el engagement etc.

Como limitación del estudio, a nivel nacional, existen pocas publicaciones científicas de medio o alto impacto relacionadas a la satisfacción laboral en centros empresariales comerciales; por ende, resulta complejo el análisis comparativo de resultados con demás segmentos; lo que existe, en ciertos repositorios, son estudios de grado sobre satisfacción, que no se encuentran indexados. Por último, la herramienta S20/23 presenta bondades con respecto a su aplicabilidad e interpretación; además, su estructura procedimental y de cuestionamientos se asemeja a condiciones de trabajo latinas, por lo que es recomendable su utilización. Esta investigación, es la primera de este tipo en este sector y puede servir de referencia para diferentes proyectos, ya que se puede profundizar el esbozo de la satisfacción con otros temas organizacionales, tales como: el liderazgo.

\section{Referencias}

Alles, M. (2015). Dirección estratégica de Recursos Humanos ( $3^{a}$ ed.). Buenos Aires: Granica.

Álvarez, L., Guzmán, M., Noda, M., Álvarez, L., y Galcerán, G. (2016). Diagnóstico de la satisfacción laboral en una entidad asistencial hospitalaria. Revista Cubana de Salud Pública, 42(3), 407-417.

Arias-Jiménez, M. (2007). Factores del clima organizacional influyentes en la satisfacción laboral de enfermería, concerniente a los cuidados intensivos neonatales del Hospital Nacional de Niños 2004. Enfermería en Costa Rica, 28(1), 12-19.

Arias, W., y Arias, G. (2014). Relación entre el clima organizacional y la satisfacción laboral en una pequeña empresa del sector privado. Ciencia \& Trabajo, 15(51), 185-191.

Arias, W., y Justo, O. (2013). Satisfacción laboral en trabajadores de dos tiendas por departamento: Un estudio comparativo. Ciencia \& Trabajo, 15(47), 41-46.

Cantón, I., y Téllez, S. (2016). La satisfacción laboral y profesional de los profesores. Revista Lasallista de Investigación, 13(1), 214-226. 
Chiang, M., Méndez, G., y Sánchez, G. (2010). Cómo influye la satisfacción laboral sobre el desempeño: Caso empresa de retail. Theoria, 19(2), 21-36.

Chiang, M., y Ojeda, J. (2013). Estudio de la relación entre satisfacción laboral y el desempeño de los trabajadores de las ferias libres. Contaduría y Administración, 58(2), 39-60.

Greenberg, J., y Baron, R. (2008). Behavior in organizations ( $9^{a}$ ed.). Texas: Pearson.

Griffin, R., Phillips, J., y Stanley, G. (2017). Comportamiento organizacional, administración de personas y organizaciones (12 $2^{a}$ ed.). México: Cengage Learning Editores S.A.

Hegney, D., Plank, A., y Parker, V. (2006). Extrinsic and intrinsic work values: Their impact on job satisfaction in nursing. Journal of Nursing Management, 14(4), 271-281.

Hernández, R., Fernández, C., y Baptista, P. (2014). Metodología de la investigación ( $6^{a}$ ed.). México: McGraw-Hill.

Manosalvas, C., Manosalvas, L., y Quintero, J. (2015). El Clima Organizacional y la Satisfacción Laboral: Un Análisis cuantitativo riguroso de su relación. Ad-minister, (26), 5-15.

Meliá, J. L., y Peiró, J. M. (1989). La medida de la satisfacción laboral en contextos organizacionales: el cuestionario de satisfacción S20/23. Psicologemas, 5, 59-74.

Meliá, J.L., y Peiró, J.M. (1998). Cuestionario de Satisfacción Laboral S20/23. Psicología de la seguridad laboral, Universidad de Valencia, 1-5.

Ministerio de Trabajo. (2005). Código de Trabajo. Quito: Lexis.

Munch, L., García, J., (2017). Fundamentos de Administración $\left(12^{a}\right.$ ed.). México: Trillas.
Patterson, M., Warr, P., y West, M. (2004). Organizational climate and company productivity: The role of employee affect and employee level. Journal of Occupational and Organizational Psychology, 77(2), 193-216.

Peña, M., Olloqui, A., y Aguilar, A. (2013). Relación de factores en la satisfacción laboral de los trabajadores de una pequeña empresa de la industria metal-mecánica. Revista Internacional Administración \& Finanzas, 6(3), 115-128.

Platán-Pérez, J., Martínez, E., y Hernández, R. (2012). El clima y la justicia organizacional y su efecto en la satisfacción laboral. Revista Internacional Administración \& Finanzas, 5(5), 1-19.

Rodríguez, A., Retamal, M., Lizana, J., y Cornejo, F. (2011). Clima y satisfacción laboral como predictores del desempeño en una organización estatal chilena. Salud \& Sociedad, 2(2), 219-234.

Salessi, S., y Omar, A. (2018). Tríada oscura de personalidad, satisfacción laboral y cinismo organizacional: un modelo estructural. Universitas Psychologica, 17(3), 21-32.

Sánchez-Sellero, M., Sánchez-Sellero, P., CruzGonzález, M., y Sánchez-Sellero, F. (2014). Características organizacionales de la satisfacción laboral en España. Revista de Administração de Empresas, 54(5), 537-547.

Sánchez-Sellero, M., y Sánchez-Sellero, P. (2018). Determinantes de la satisfacción laboral en la industria de la madera y el papel: Estudio en España y hallazgos en otros países. Maderas. Ciencia y tecnología, 20(4), 641-660.

Silva, M. (1996). El clima en las organizaciones. Teoría, método e intervención ( $1^{a}$ ed.). Barcelona: EUB, S.L. 
Temkin, B., y Cruz, J. (2018). Las dimensiones de la actividad laboral y la satisfacción con el trabajo y con la vida: el caso de México. Estudios Sociológicos, 36(108), 507-538.

Weinert, A. (1985). Manual de psicología de la organización ( $1^{a}$ ed.). Barcelona: Herder.

Yohalmo, R. (2014). Capacitar o morir: la capacitación de personal como estrategia competitiva ( $1^{a}$ ed.). San Salvador: León Editores.

Zayas, P., Báez, R., Zayas, J., y Hernández, M. (2015). Causas de la satisfacción laboral en una organización comercializadora mayorista. Revista Facultad de Ciencias Económicas: Investigación y Reflexión, 23(2), 35-51. 